\section{Uso de serviços de saúde por diabéticos cobertos por plano privado em comparação aos usuários do Sistema Único de Saúde no Município de Belo Horizonte, Minas Gerais, Brasil}

\author{
Use of health services by diabetics with private \\ health insurance compared to users of the Brazilian \\ Unified National Health System in Belo Horizonte, \\ Minas Gerais State, Brazil
}

\author{
Uso de servicios de salud por parte de diabéticos \\ cubiertos por plan privado, en comparación con \\ los usuarios del Sistema Único de Salud en el \\ municipio de Belo Horizonte, Minas Gerais, Brasil
}

Sara de Souza Silva 1

Juliana Vaz de Melo Mambrini 1,2

Maria Aparecida Turci 2

James Macinko ${ }^{3}$

Maria Fernanda Lima-Costa 1,2

\title{
Resumo
}

O objetivo do trabalho foi comparar o uso de serviços de saúde, o uso de medicamentos, a cobertura de exames de rastreamento, os atributos da atenção primária em saúde e os comportamentos em saúde de indivíduos ( $n=370$ ) com 45 anos ou mais, com diagnóstico médico de diabetes, segundo as fontes de atenção em saúde, assim definidas: afiliados a plano privado de saúde, independentemente do local de residência; residentes em áreas cobertas pela Estratégia Saúde da Família (ESF); cobertos pela unidade básica de saúde (UBS) "tradicional". O estudo utilizou os dados do 2o Inquérito de Saúde de Adultos da Região Metropolitana de Belo Horizonte (2010). Os indicadores de uso de serviços de saúde, o uso de medicamentos, a cobertura de exames de acompanhamento, o consumo abusivo de álcool e o tabagismo atual não diferiram significativamente entre as fontes de atenção. As prevalências da prática insuficiente de exercícios físicos no tempo livre e do sedentarismo no cotidiano foram maiores entre os cobertos pela ESF ou UBS "tradicional". Os atributos da atenção primária em saúde (acesso, integralidade, longitudinalidade e foco familiar) apresentaram melhor desempenho entre os afiliados a plano privado de saúde, mesmo após ajuste por idade, sexo e escolaridade. Os resultados da investigação apontam dois principais desafios para o Sistema Único de Saúde no manejo dos pacientes com diabetes no Município de Belo Horizonte, Brasil: a realização de ações mais efetivas para a promoção da atividade física e a necessidade de reorganização visando à melhoria no desempenho da atenção primária, sobretudo no acesso às consultas médicas.

Diabetes Mellitus; Serviços de Saúde; Saúde da Família; Atenção Primária à Saúde

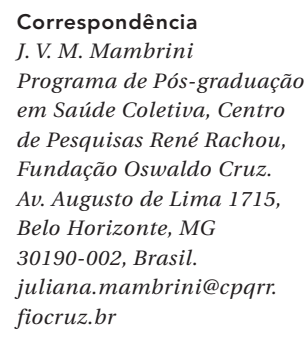




\section{Introdução}

As doenças e agravos não transmissíveis (DANT) são consideradas, pela Organização das Nações Unidas, uma epidemia em ascensão em países de alta, média e baixa renda 1 . Entre as 57 milhões de mortes ocorridas ao redor do mundo em 2008, $63 \%$ foram devidas às DANT, particularmente doenças cardiovasculares, diabetes, cânceres e doenças crônicas respiratórias ${ }^{1}$. Globalmente, os principais fatores de risco para a mortalidade são o aumento da pressão arterial (13\% das mortes), o consumo do tabaco (9\%), a hiperglicemia (6\%), a inatividade física (6\%) e a obesidade (5\%) 2. Populações com baixo nível socioeconômico são mais vulneráveis às DANT, devido principalmente à associação inversa entre a prevalência dos seus fatores de risco e o nível de escolaridade 3 .

A população brasileira tem vivido uma rápida transição demográfica, epidemiológica e nutricional 4. A transição nutricional caracteriza-se pelo atual predomínio de dieta baixa em fibras e com altos níveis de consumo de açúcar e de ácidos graxos saturados, associada à alta prevalência de sedentarismo, fatores estes que contribuem para o aumento da incidência do diabetes do tipo II ${ }^{5}$. Nas duas últimas décadas, a mortalidade por diabetes na população brasileira, com idades entre 30 e 70 anos, aumentou $8 \%$ 6. Entre os idosos (60 anos ou mais), esse aumento foi maior, correspondendo a $18 \%$ 7. O Ministério da Saúde considera que os "hábitos de vida saudáveis são a base do tratamento do diabetes, sobre o qual pode ser acrescido - ou não - o tratamento farmacológico" 8 (p. 49). Esses hábitos incluem a atividade física regular, a alimentação saudável e o controle do peso, além de evitar o fumo e o consumo excessivo de bebidas alcoólicas 8,9.

Cerca de 9\% dos gastos com internações hospitalares na rede pública brasileira são atribuídos ao diabetes 10 . Parcela importante das complicações da doença pode ser evitada por meio da atenção primária oportuna e de qualidade. No seu conjunto (códigos da 10a revisão da Classificação Internacional de Doenças: E-10 a E-14), cerca de 120 mil hospitalizações na rede pública (ou seja, 6,5 internações por 10 mil habitantes) poderiam ter sido evitadas, no ano de 2006, com ações efetivas no âmbito da atenção primária no Brasil 11. De acordo com o marco teórico de Starfield 12 , a atenção primária, para ser efetiva, deve basear-se em um modelo que visa ao cuidado longitudinal, à atenção integral e à provisão de cuidados no contexto da família e da comunidade, além da coordenação dos diferentes níveis de atenção. A atenção primária no Brasil tem expandido consideravelmente por meio da Estratégia Saúde da Família (ESF) 13.
Bertoldi et al. 4, em recente revisão da literatura sobre diabetes no Brasil, chamaram a atenção para a raridade de estudos que examinam diferenças na qualidade do tratamento de pacientes com diabetes no Sistema Único de Saúde (SUS) em comparação a planos privados de saúde. No nosso conhecimento, até a presente data, a única investigação sobre o tema no nosso meio é um estudo de coorte retrospectiva, comparando 80 pacientes atendidos em ambulatórios do SUS e 277 pacientes em clínicas privadas 14 . Os resultados mostraram pior controle metabólico entre os primeiros ${ }^{14}$, mas a generalização desses resultados é questionada devido à sua circunscrição local e pequeno número de participantes 5 .

O presente trabalho, conduzido no Município de Belo Horizonte, Minas Gerais, tem por objetivo comparar indicadores de uso de serviços de saúde e comportamentos em saúde entre indivíduos que sabem ser portadores de diabetes e que são afiliados a planos privados de saúde em relação aos seus pares usuários do sistema público de saúde.

\section{Métodos}

\section{Área de estudo}

Belo Horizonte, capital do Estado de Minas Gerais, é uma das maiores cidades brasileiras, com 2,4 milhões de habitantes 15. A Secretaria Municipal de Saúde de Belo Horizonte (SMS-BH) desenvolveu um índice de vulnerabilidade em saúde (IVS), baseado no perfil da mortalidade, nas condições socioeconômicas dos moradores e nas condições de saneamento dos setores censitários do Instituto Brasileiro de Geografia e Estatística (IBGE). As áreas consideradas como de média e alta vulnerabilidades foram selecionadas para a implantação da ESF a partir de 2003. No período compreendido entre 2003 e 2010, houve um aumento no número de equipes da ESF, alcançando a cobertura de $71 \%$ do total da população adulta no ano final, quando todas as unidades básicas de saúde (UBS) "tradicionais" nas áreas de cobertura da ESF foram substituídas pelo novo modelo de atenção, permanecendo na modalidade "tradicional” de atenção à saúde as áreas classificadas como de baixo risco 16. Em 2010, 46,7\% da população com 20 anos ou mais eram afiliadas a planos privados de saúde 16 .

\section{Fonte de dados}

Os dados utilizados para este trabalho foram aqueles do 20 Inquérito de Saúde de Adultos da Região Metropolitana de Belo Horizonte, realiza- 
do entre maio e julho de 2010. Os participantes do inquérito foram selecionados por meio de amostra probabilística de conglomerados, estratificada em dois estágios. Os setores censitários do IBGE foram usados como unidade de seleção e a unidade amostral foi o domicílio. A amostra foi baseada em 7.500 domicílios, dos quais $77,3 \%$ participaram do inquérito. Todos os cerca de 8 mil residentes nos domicílios amostrados, com idade igual ou superior a 20 anos, foram elegíveis para a entrevista face a face. O inquérito de saúde foi aprovado pelo Comitê de Ética do Centro de Pesquisas René Rachou, Fundação Oswaldo Cruz (carta de aprovação no 10/2009). Mais detalhes estão disponíveis em publicações anteriores 7,16.

População do estudo, definição de diabetes e classificação das fontes de atenção à saúde

Para o presente trabalho foram selecionados todos os participantes do inquérito, residentes no Município de Belo Horizonte, com idade igual ou superior a 45 anos, e que informaram ser portadores de diabetes. Essa faixa etária foi escolhida por ser considerada a de maior risco para o diabetes ${ }^{8}$. A condição de ser portador dessa doença foi definida pela história de diagnóstico médico, por meio da pergunta geral "algum médico já disse que você tem diabetes?”.

A fonte da atenção à saúde foi definida com base na localização dos domicílios e na informação individual de ser afiliado a plano de saúde. Todos os domicílios participantes da pesquisa foram georreferenciados por setor censitário. Com base nessa localização, os participantes do estudo foram classificados em cobertos pela ESF ou pela UBS “tradicional”, considerando-se os parâmetros estabelecidos pela SMS-BH, anteriormente mencionados 16 . O uso exclusivo do SUS foi atribuído àqueles que informaram não ser afiliados a plano privado de saúde. Assim, a população do estudo foi classificada nos seguintes grupos: (1) afiliados a plano privado de saúde, independentemente do local de residência; (2) não possuem plano privado de saúde e são residentes em áreas cobertas pela ESF; (3) cobertos pela UBS “tradicional" (não têm plano privado de saúde e são residentes em áreas não cobertas pela ESF).

\section{Outras variáveis do estudo}

As variáveis dependentes do estudo foram os indicadores de uso de serviços de saúde e de medicamentos, incluindo dentre estes a realização de exames de rastreamento para o controle do risco cardiovascular e do diabetes e os atributos da atenção primária, alguns comportamentos em saúde, assim como aconselhamento sobre hábitos saudáveis. $\mathrm{O}$ aconselhamento sobre hábitos saudáveis e os comportamentos em saúde considerados na presente análise incluíram atividades físicas no lazer, atividades físicas no cotidiano, tabagismo atual e consumo abusivo de álcool.

Entre os indicadores de uso de serviços de saúde e de medicamentos foram considerados a existência de fonte regular de cuidados (em caso de necessidade costuma procurar o mesmo serviço ou profissional de saúde), a realização de pelo menos uma consulta médica nos 12 meses precedentes, o uso regular de medicamentos e a existência de dificuldades para a obtenção dos medicamentos de uso regular (devido a problemas financeiros, dificuldades em encontrar o medicamento e dificuldades em se deslocar para obtê-lo, entre outras).

Entre os exames de rastreamento, foram considerados: aferição da pressão arterial uma ou mais vezes nos últimos 12 meses; aferição do colesterol uma ou mais vezes nos últimos 12 meses 8 ; e aferição da glicemia uma ou mais vezes nos últimos 12 meses.

Os atributos da atenção primária considerados na presente análise foram baseados no marco teórico de Starfield 12, levando-se em conta: o primeiro contato (precisa consultar um médico generalista para ser encaminhado a um especialista); o acesso (não tem queixas para conseguir consultas médicas quando precisa, considerando-se como queixa a existência de filas, a falta de médicos no local do atendimento, as dificuldades de deslocamento para este local, as dificuldades financeiras etc.); integralidade (quem lhe atende é capaz de resolver a maioria dos seus problemas de saúde); longitudinalidade (é atendido pelo mesmo profissional a cada nova consulta); foco familiar (quem lhe atende pergunta sobre os problemas de saúde da sua família). Essas informações foram obtidas para os participantes do inquérito de saúde que informaram ter profissional ou serviço de saúde que costuma procurar.

Com referência ao aconselhamento sobre hábitos saudáveis, foram considerados o recebimento de aconselhamento médico, alguma vez na vida, sobre o uso de tabaco, atividades físicas, hábitos alimentares e/ou uso de álcool. Os comportamentos em saúde incluídos na análise foram: atividade física no lazer, atividade física no cotidiano, tabagismo e o consumo abusivo de álcool. A atividade física no lazer foi definida pela frequência semanal de atividades de intensidade leve, moderada ou intensa (caminhada para fazer exercícios, ginástica ou prática de esportes, entre outras) por pelo menos 30 minutos diários, nos últimos 90 dias; foi considerada regular a atividade física três ou mais dias na semana. $\mathrm{O}$ 
sedentarismo no cotidiano foi atribuído àqueles que informaram ficar geralmente sentado durante o dia e andar pouco; o não sedentarismo foi atribuído àqueles que informaram ficar em pé ou caminhar bastante durante o dia e carregar peso de vez em quando e/ou levantar/carregar volumes (caixas, livros, entre outros) ou geralmente subir escadas ou ladeiras e/ ou fazer trabalho pesado, tendo de carregar volumes pesados (caixas, livros, tijolos, sacos de cimento etc.). O hábito de fumar foi definido pelo consumo atual de cigarros entre aqueles que já fumaram pelo menos cem cigarros durante toda a vida. O consumo abusivo de bebidas alcoólicas foi atribuído àqueles que consumiram 5 ou mais doses de álcool em uma única ocasião nos últimos 30 dias. Idade (variável contínua), sexo e escolaridade completa (<4, 4-7 e 8 anos ou mais) foram considerados a priori potenciais variáveis de confusão no estudo.

\section{Análise estatística}

Os testes do qui-quadrado e a análise de variância foram utilizados nas análises não ajustadas para examinar diferenças entre frequências e médias, respectivamente. As análises multivariadas foram baseadas em razões de prevalência, estimadas por meio da regressão de Poisson com variância robusta para eventos comuns 17 . Para cada variável dependente foram construídos dois modelos multivariados: no primeiro, as estimativas foram ajustadas por sexo e idade; no segundo modelo, as estimativas foram adicionalmente ajustadas pelo nível de escolaridade.

As análises foram realizadas usando-se a versão 13.0 do pacote estatístico Stata (StataCorp LP, College Station, Estados Unidos), incorporandose os pesos individuais, o delineamento complexo da amostra e o efeito da agregação domiciliar.

\section{Resultados}

Dos 3.473 participantes do 2o Inquérito de Saúde de Adultos da Região Metropolitana de Belo Horizonte com 45 anos ou mais de idade, 370 (10,7\%) haviam tido diagnóstico médico de diabetes e participaram da presente análise. A prevalência dos que informaram ser diabéticos não diferiu significativamente entre as diferentes fontes de atenção: 10,6\% (IC95\%: 9,2\%-12,2\%) entre afiliados a plano privado de saúde, $11,4 \%$ (IC95\%: 9,8\%-13,2\%) entre residentes em áreas cobertas pela ESF e 8,9\% (IC95\%: 6,6\%-11,9\%) entre os cobertos pela UBS “tradicional” ( $p=0,325)$.

Como pode ser visto na Tabela 1 , a média da idade dos participantes foi igual a 64,8 anos. En- tre esses, predominou o sexo feminino $(58,1 \%)$ e a escolaridade igual a $4-7(37,2 \%)$ ou 8 anos ou mais $(40,2 \%)$. A média da idade foi mais alta entre afiliados a plano privado de saúde $(66,4$ anos) em comparação aos cobertos pela ESF (62,8 anos) ou pela UBS “tradicional” (64,3 anos) $(\mathrm{p}=0,037)$. A distribuição por gênero não variou significativamente entre as fontes de atenção $(p=0,914)$. Por outro lado, observou-se grande heterogeneidade no nível de escolaridade ( $\mathrm{p}<0,001)$ : a prevalência da escolaridade igual ou superior a 8 anos foi cerca de duas vezes maior entre afiliados a plano privado (56\%) em comparação aos cobertos pela ESF $(22,7 \%)$ ou pela UBS “tradicional” (29,1\%).

A distribuição dos indicadores de uso de serviços de saúde e de medicamentos por fonte da atenção está apresentada na Tabela 2 . O relato de ter profissional ou serviço de saúde que costuma procurar em caso de necessidade não diferiu entre as fontes de atenção ( $\mathrm{p}=0,612)$, variando entre $96 \%$ nos afiliados a plano privado, $97 \%$ nos cobertos pela ESF e $93,5 \%$ nos cobertos pela UBS “tradicional”. A proporção dos que haviam realizado uma ou mais consultas médicas nos 12 meses precedentes não diferiu significativamente entre afiliados a plano privado $(92,2 \%)$ e cobertos pela ESF $(87,3 \%)$ ou pela UBS “tradicional” $(82,6 \%)(p=0,179)$. O uso regular de medicamentos não diferiu significativamente $(\mathrm{p}=0,250)$ entre afiliados a plano privado $(90,7 \%)$, cobertos pela ESF $(83,9 \%)$ e cobertos pela UBS "tradicional” (87,9\%). A proporção dos que informaram não ter dificuldade para a obtenção de medicamentos de uso regular também não diferiu significativamente $(\mathrm{p}=0,271)$ entre as diferentes fontes de atenção, variando entre $82,4 \%$ dentre afiliados a plano privado, $73 \%$ pelos cobertos pela ESF e 76,9\% cobertos pela outra fonte. As semelhanças anteriormente comentadas persistiram nos modelos ajustados por sexo e idade, assim como naqueles ajustados adicionalmente por nível de escolaridade.

Na Tabela 3, observa-se que as coberturas dos exames para aferição da glicemia, da pressão arterial e do colesterol foram altas em todas as fontes de atenção. A proporção dos que haviam tido a glicemia aferida pelo menos uma vez nos últimos 12 meses foi igual a $97,8 \%$ entre os afiliados a plano privado, $96,5 \%$ dentre os cobertos pela ESF e 93,4\% entre os cobertos pela UBS “tradicional” ( $p=0,426)$. As proporções correspondentes para aferição da hipertensão arterial nos últimos 12 meses foram de 96,8\%, 97,9\% e 100\%, respectivamente $(\mathrm{p}=0,536)$. A proporção dos que aferiram o seu colesterol pelo menos uma vez nos últimos 12 meses foi significativamente mais alta entre os afiliados a plano privado $(96,6 \%)$ e os cobertos pela ESF $(97,9 \%)$ em comparação 
Tabela 1

Características sociodemográficas dos participantes com 45 anos ou mais de idade com diagnóstico médico de diabetes, segundo a fonte de atenção em saúde. Município de Belo Horizonte, Minas Gerais, Brasil, 2010.

\begin{tabular}{|c|c|c|c|c|c|}
\hline \multirow[t]{2}{*}{ Características sociodemográficas } & \multirow[t]{2}{*}{ Total } & \multicolumn{3}{|c|}{ Fonte de atenção } & \multirow[t]{2}{*}{ Valor de $p$} \\
\hline & & Plano privado & ESF & UBS "tradicional" & \\
\hline Idade em anos [média (DP)] & $64,8(10,9)$ & $66,4(10,9)$ & $62,8(10,5)$ & $64,3(11,3)$ & 0,037 * \\
\hline Sexo feminino [\%] & 58,1 & 56,9 & 59,4 & 59,0 & $0,914 * \star$ \\
\hline Escolaridade em anos completos [\%] & & & & & $<0,001$ ** \\
\hline$<4$ & 22,6 & 12,4 & 33,8 & 30,8 & \\
\hline $4-7$ & 37,2 & 31,7 & 43,5 & 40,1 & \\
\hline $8+$ & 40,2 & 56,0 & 22,7 & 29,1 & \\
\hline Número de entrevistados & 370 & 178 & 152 & 40 & - \\
\hline
\end{tabular}

DP: desvio padrão; ESF: Estratégia Saúde da Família; UBS: unidade básica de saúde.

* Teste de variância para diferenças entre as fontes;

** Teste do qui-quadrado para diferença entre as fontes.

Tabela 2

Indicadores de uso de serviços de saúde e de medicamentos entre participantes com 45 anos ou mais de idade com diagnóstico médico de diabetes segundo a fonte da atenção em saúde. Município de Belo Horizonte, Minas Gerais, Brasil, 2010.

\begin{tabular}{|c|c|c|c|c|}
\hline Fonte de atenção & $\begin{array}{l}\text { Possui fonte regular } \\
\text { de cuidados em } \\
\text { saúde }\end{array}$ & $\begin{array}{l}\text { Realizou pelo menos } \\
\text { uma consulta médica } \\
\text { nos últimos } 12 \text { meses }\end{array}$ & $\begin{array}{l}\text { Faz uso regular de } \\
\text { medicamentos }\end{array}$ & $\begin{array}{c}\text { Relata não ter } \\
\text { dificuldade } \\
\text { quando precisa de } \\
\text { medicamentos de uso } \\
\text { regular }\end{array}$ \\
\hline \multicolumn{5}{|l|}{ Proporções (IC95\%) } \\
\hline Plano privado & $96,0(92,7-99,3)$ & $92,2(88,2-96,2)$ & $90,7(85,8-95,5)$ & $82,4(74,5-90,3)$ \\
\hline ESF & $97,0(94,2-99,7)$ & $87,3(81,7-92,9)$ & $83,9(77,4-90,4)$ & $73,0(64,2-81,9)$ \\
\hline UBS "tradicional" & $93,5(86,0-100,9)$ & $82,6(68,5-96,7)$ & $87,9(76,3-99,5)$ & $76,9(61,5-92,3)$ \\
\hline Valor de $p$ * & 0,612 & 0,179 & 0,250 & 0,271 \\
\hline \multicolumn{5}{|c|}{ RP (IC95\%) ajustadas por idade e sexo } \\
\hline Plano privado & 1,00 & 1,00 & 1,00 & 1,00 \\
\hline ESF & $1,01(0,97-1,06)$ & $0,94(0,87-1,02)$ & $0,94(0,85-1,03)$ & $0,89(0,75-1,04)$ \\
\hline UBS "tradicional" & $0,97(0,89-1,06)$ & $0,89(0,74-1,07)$ & $0,98(0,85-1,12)$ & $0,93(0,75-1,16)$ \\
\hline \multicolumn{5}{|c|}{$\begin{array}{l}\text { RP (IC95\%) ajustadas por idade, sexo e } \\
\text { escolaridade }\end{array}$} \\
\hline Plano privado & 1,00 & 1,00 & 1,00 & 1,00 \\
\hline ESF & $1,01(0,96-1,06)$ & $0,94(0,86-1,02)$ & $0,95(0,87-1,03)$ & $0,94(0,79-1,11)$ \\
\hline UBS "tradicional" & $0,97(0,89-1,06)$ & $0,89(0,75-1,06)$ & $0,99(0,86-1,13)$ & $0,99(0,80-1,24)$ \\
\hline Número de entrevistados & 370 & 370 & 370 & 325 ** \\
\hline
\end{tabular}

ESF: Estratégia Saúde da Família; IC95\%: intervalo de 95\% de confiança; RP: razões de prevalência; UBS: unidade básica de saúde.

Nota: as RPs e respectivos IC95\% foram estimados por meio de regressão de Poisson com variância robusta, considerando-se os parâmetros amostrais.

* Teste qui-quadrado para diferenças entre as fontes de atenção;

** Somente aqueles que informaram consumir medicamentos de uso regular.

aos cobertos pela UBS “tradicional” $(84,6 \%)(\mathrm{p}=$ 0,004), mas esta diferença desapareceu após o ajuste por sexo e idade. Os demais resultados não foram modificados após esses ajustes. Com relação aos aconselhamentos, observa-se que a pro- porção dos que receberam orientações médicas acerca de hábitos saudáveis foi alta em todos os grupos $(94,5 \%$ dos afiliados a plano privado, $89 \%$ dos cobertos pela ESF e $91,2 \%$ dos cobertos pela UBS “tradicional”; $\mathrm{p}=0,360$ ). 
Tabela 3

Aconselhamento sobre hábitos saudáveis e aferição da glicemia, da pressão arterial e do colesterol entre participantes com 45 anos ou mais de idade com diagnóstico médico de diabetes, segundo a fonte da atenção em saúde. Município de Belo Horizonte, Minas Gerais, Brasil, 2010.

\begin{tabular}{|c|c|c|c|c|}
\hline Fonte de atenção & $\begin{array}{c}\text { Aferição da } \\
\text { glicemia nos } \\
\text { últimos } 12 \text { meses }\end{array}$ & $\begin{array}{l}\text { Aferição da pressão } \\
\text { arterial nos últimos } \\
12 \text { meses }\end{array}$ & $\begin{array}{l}\text { Aferição do } \\
\text { colesterol nos } \\
\text { últimos } 12 \text { meses }\end{array}$ & $\begin{array}{l}\text { Recebeu aconselhamento } \\
\text { médico alguma vez na vida } \\
\text { sobre tabagismo, consumo } \\
\text { de álcool, atividades físicas } \\
\text { e/ou hábitos alimentares }\end{array}$ \\
\hline \multicolumn{5}{|l|}{ Proporções (IC95\%) } \\
\hline Plano privado & $97,8(95,6-100,0)$ & $96,8(93,9-99,6)$ & $96,6(93,9-99,3)$ & $94,5(89,1-100,0)$ \\
\hline ESF & $96,5(93,4-99,6)$ & $97,9(95,5-100,4)$ & $97,9(95,5-100,4)$ & $89,0(83,4-94,7)$ \\
\hline UBS "tradicional" & $93,4(83,9-102,9)$ & 100,0 & $84,6(70,0-99,2)$ & $91,2(81,7-100,7)$ \\
\hline Valor de $p$ * & 0,426 & 0,536 & 0,004 & 0,360 \\
\hline \multicolumn{5}{|c|}{ RP (IC95\%) ajustadas por idade e sexo } \\
\hline Plano privado & 1,00 & 1,00 & 1,00 & 1,00 \\
\hline ESF & $0,99(0,94-1,03)$ & $1,01(0,97-1,05)$ & $1,02(0,97-1,06)$ & $0,93(0,87-1,00)$ \\
\hline UBS "tradicional" & $0,95(0,86-1,06)$ & $1,03(1,00-1,06)$ & $0,88(0,74-1,05)$ & $0,96(0,86-1,07)$ \\
\hline \multicolumn{5}{|c|}{$\begin{array}{l}\text { RP (IC95\%) ajustadas por idade, sexo e } \\
\text { escolaridade }\end{array}$} \\
\hline Plano privado & 1,00 & 1,00 & 1,00 & 1,00 \\
\hline ESF & $0,99(0,93-1,05)$ & $1,02(0,96-1,08)$ & $1,03(0,97-1,10)$ & $0,95(0,89-1,02)$ \\
\hline UBS "tradicional" & $0,96(0,86-1,07)$ & $1,04(0,99-1,09)$ & $0,89(0,75-1,06)$ & $0,98(0,87-1,10)$ \\
\hline Número de entrevistados & 370 & 370 & 370 & 370 \\
\hline
\end{tabular}

ESF: Estratégia Saúde da Família; IC95\%: intervalo de 95\% de confiança; RP: razões de prevalência; UBS: unidade básica de saúde.

Nota: as RPs e respectivos IC95\% foram estimados por meio de regressão de Poisson com variância robusta, considerando-se os parâmetros amostrais.

* Teste qui-quadrado para diferenças entre as fontes de atenção.

O desempenho da atenção primária foi avaliado e diferiu nas várias fontes de atenção (Tabela 4). Entre os cobertos pela ESF e pela UBS "tradicional" predominou a necessidade de consulta com médico generalista antes da consulta com o especialista (81,8\% e 93,9\%, respectivamente), ao passo que entre os afiliados a plano privado, menos da metade $(47,1 \%)$ precisava consultar um médico generalista antes da consulta com o especialista $(\mathrm{p}<0,001)$. O acesso, definido pela ausência de dificuldades para conseguir consultas médicas, foi significativamente mais alto entre os afiliados a plano privado (65,6\% informaram não ter dificuldades) em comparação aos cobertos pela ESF $(22,6 \%)$ e aos atendidos na UBS "tradicional” $(21,9 \%)(p<0,001)$. A integralidade, na percepção do usuário, foi mais alta entre os afiliados a plano privado em comparação aos atendidos pela ESF e pela UBS “tradicional” (89,2\%, $74,3 \%$ e $73,6 \%$, respectivamente, consideravam que quem lhes atendia era capaz de resolver a maioria dos seus problemas de saúde; $p=0,004$ ). A longitudinalidade também foi mais frequente entre os afiliados a plano privado em relação aos cobertos pela ESF e pela UBS "tradicional"
(86,3\%, 69,6\% e 74,7\%, respectivamente, informaram ser atendidos pelo mesmo profissional a cada nova consulta; $p=0,005$ ). $O$ enfoque familiar foi informado com menos frequência pelos cobertos pela ESF em comparação aos afiliados a plano privado e pela UBS “tradicional" $(65,3 \%$, $89,4 \%$ e $82,9 \%$, respectivamente, informaram que quem lhes atendia perguntava pelas condições de saúde de outros membros da família; $\mathrm{p}<$ $0,001)$. Via de regra, essas diferenças persistiram nas análises ajustadas por idade, sexo e adicionalmente por escolaridade.

Na Tabela 5, está apresentada a distribuição de alguns comportamentos em saúde, segundo a fonte de atenção. A prevalência da atividade física regular no tempo livre foi significativamente mais alta entre afiliados a plano privado de saúde $(18,7 \%)$ em comparação aos cobertos pela ESF $(7,2 \%)$ e pela UBS “tradicional” $(8,4 \%)$; após ajustes por idade, sexo e escolaridade, os cobertos pela ESF persistiram menos propensos a realizar atividade física regular no tempo livre em comparação àqueles afiliados a plano privado (RP = 0,46; IC95\%: 0,22-0,95). A prevalência do sedentarismo no cotidiano variou entre $52,1 \%$ 
Atributos da atenção primária nos serviços de saúde utilizados por participantes com 45 anos ou mais de idade com diagnóstico médico de diabetes, segundo a fonte da atenção em saúde. Município de Belo Horizonte, Minas Gerais, Brasil, 2010.

\begin{tabular}{|c|c|c|c|c|c|}
\hline Fonte de atenção & $\begin{array}{c}\text { Primeiro contato } \\
\text { (precisa consultar } \\
\text { um médico } \\
\text { generalista para ser } \\
\text { encaminhado a um } \\
\text { especialista) }\end{array}$ & $\begin{array}{l}\text { Acesso (relata não } \\
\text { ter dificuldade } \\
\text { para conseguir } \\
\text { consulta médica } \\
\text { quando precisa) }\end{array}$ & $\begin{array}{c}\text { Integralidade } \\
\text { (considera que } \\
\text { quem lhe atende é } \\
\text { capaz de resolver } \\
\text { a maioria dos seus } \\
\text { problemas de } \\
\text { saúde) }\end{array}$ & $\begin{array}{l}\text { Longitudinali- } \\
\text { dade (informa ser } \\
\text { atendido pelo } \\
\text { mesmo profissional } \\
\text { a cada nova } \\
\text { consulta) }\end{array}$ & $\begin{array}{c}\text { Foco familiar } \\
\text { (considera que } \\
\text { quem lhe atende } \\
\text { pergunta sobre os } \\
\text { problemas de saúde } \\
\text { da sua família) }\end{array}$ \\
\hline \multicolumn{6}{|l|}{ Proporções (IC95\%) } \\
\hline Plano privado & $47,1(37,9-56,2)$ & $65,6(56,9-74,4)$ & $89,2(84,5-94,0)$ & $86,3(80,7-91,9)$ & $89,4(83,6-95,1)$ \\
\hline ESF & $81,8(74,9-88,7)$ & $22,6(14,9-30,3)$ & $74,3(65,9-82,7)$ & $69,6(61,2-78,0)$ & $65,3(56,4-74,1)$ \\
\hline UBS "tradicional" & $93,9(85,1-102,7)$ & $21,9(6,0-37,7)$ & $73,6(57,5-89,8)$ & $74,7(59,0-90,4)$ & $82,9(71,2-94,5)$ \\
\hline Valor de $p$ * & $<0,001$ & $<0,001$ & 0,004 & 0,005 & $<0,001$ \\
\hline \multicolumn{6}{|c|}{ RP (IC95\%) ajustadas por idade e sexo } \\
\hline Plano privado & 1,00 & 1,00 & 1,00 & 1,00 & 1,00 \\
\hline ESF & $1,74 * \star(1,41-2,15)$ & $0,36 * \star(0,25-0,51)$ & $0,84 * \star(0,74-0,95)$ & $0,80 * \star(0,70-0,92)$ & $0,73 * \star(0,63-0,86)$ \\
\hline UBS "tradicional" & $2,01 * \star(1,62-2,48)$ & $0,34 * \star(0,16-0,72)$ & $0,83(0,66-1,03)$ & $0,86(0,69-1,08)$ & $0,93(0,80-1,08)$ \\
\hline \multicolumn{6}{|c|}{$\begin{array}{l}\text { RP (IC95\%) ajustadas por idade, sexo e } \\
\text { escolaridade }\end{array}$} \\
\hline Plano privado & 1,00 & 1,00 & 1,00 & 1,00 & 1,00 \\
\hline ESF & $1,66 * *(1,32-2,08)$ & $0,41 * \star(0,28-0,59)$ & $0,87 * \star(0,77-0,99)$ & $0,83 * \star(0,72-0,97)$ & $0,77 * \star(0,65-0,90)$ \\
\hline UBS "tradicional" & $1,93 * \star(1,54-2,42)$ & $0,43 * \star(0,22-0,81)$ & $0,86(0,68-1,08)$ & $0,89(0,71-1,12)$ & $0,96(0,83-1,12)$ \\
\hline Número de entrevistados & $355^{\star \star \star}$ & $355^{\star \star \star \star}$ & $355^{\star \star \star \star}$ & $355^{\star \star \star}$ & $355 * \star \star$ \\
\hline
\end{tabular}

ESF: Estratégia Saúde da Família; IC95\%: intervalo de 95\% de confiança; RP: razões de prevalência; UBS: unidade básica de saúde.

Nota: as RPs e respectivos IC95\% foram estimados por meio de regressão de Poisson com variância robusta, considerando-se os parâmetros amostrais.

* Teste qui-quadrado para diferenças entre as fontes de atenção;

$\star \star p<0,05$;

*** Somente aqueles que informaram possuir fonte regular de cuidados.

nos afiliados a plano privado, $50,8 \%$ nos cobertos pela ESF e 70,8\% nos cobertos pela UBS "tradicional” ( $\mathrm{p}=0,107$ ); após ajustes por idade, sexo e escolaridade, os cobertos pela UBS "tradicional" apresentaram maior propensão ao sedentarismo $(\mathrm{RP}=1,35$; IC95\%: 1,04-1,77). A prevalência do consumo abusivo de álcool não diferiu significativamente dentre os cobertos por plano privado, ESF ou UBS “tradicional” (24,4\%, 17,7\% e $21,2 \%$, respectivamente; $\mathrm{p}=0,403$ ), assim como a prevalência do tabagismo atual (7,1\%, 10,6\% e 12,8\%, respectivamente; $\mathrm{p}=0,375)$; estas semelhanças não foram afetadas pelos ajustes por idade, sexo e escolaridade.

\section{Discussão}

Os resultados deste trabalho, baseado em inquérito de base populacional de pessoas com diagnóstico médico de diabetes, mostram que existem diferenças importantes no desempenho de alguns atributos da atenção primária, segundo a fonte de atenção à saúde. De forma geral, esses atributos apresentaram melhor desempenho entre afiliados a plano privado de saúde, em comparação aos usuários exclusivos do SUS. Por outro lado, os indicadores de uso de serviços e de medicamentos, a cobertura de exames preventivos e os comportamentos prejudiciais à saúde (com exceção de atividades físicas) foram semelhantes entre indivíduos atendidos pelas diferentes fontes de atenção à saúde.

A abordagem do diabético na atenção primária em saúde compreende a promoção da saúde (abordagem dos fatores de risco), a detecção precoce (exames de rastreamento), o tratamento e o acompanhamento, a coordenação do cuidado (sistema de referência e contrarreferência) e o manejo de complicações da doença ${ }^{8}$. O Ministério da Saúde estabelece alguns indicadores para a abordagem de pessoas com a doença, que 
Comportamentos em saúde entre participantes com 45 anos ou mais de idade com diagnóstico médico de diabetes, segundo a fonte da atenção em saúde. Município de Belo Horizonte, Minas Gerais, Brasil, 2010.

\begin{tabular}{|c|c|c|c|c|}
\hline Fonte de atenção & $\begin{array}{l}\text { Atividade física } \\
\text { regular no tempo } \\
\text { livre }\end{array}$ & $\begin{array}{c}\text { Sedentarismo no } \\
\text { cotidiano }\end{array}$ & $\begin{array}{c}\text { Consumo abusivo de } \\
\text { álcool }\end{array}$ & Tabagismo atual \\
\hline \multicolumn{5}{|l|}{ Proporções (IC95\%) } \\
\hline Plano privado & $18,7(12,3-25,0)$ & $52,1(43,3-60,9)$ & $24,4(17,6-31,2)$ & $7,1(3,1-11,0)$ \\
\hline ESF & $7,2(3,0-11,4)$ & $50,8(42,2-59,5)$ & $17,7(11,1-24,2)$ & $10,6(5,5-15,7)$ \\
\hline UBS "tradicional" & $8,4(0,0-18,9)$ & $70,8(55,9-85,6)$ & $21,2(7,1-35,2)$ & $12,8(2,9-22,8)$ \\
\hline Valor de $p$ * & 0,020 & 0,107 & 0,403 & 0,375 \\
\hline \multicolumn{5}{|c|}{ RP (IC95\%) ajustadas por idade e sexo } \\
\hline Plano privado & 1,00 & 1,00 & 1,00 & 1,00 \\
\hline ESF & $0,38 * \star(0,19-0,74)$ & $1,05(0,83-1,32)$ & $0,71(0,46-1,10)$ & $1,28(0,60-2,71)$ \\
\hline UBS "tradicional" & $0,45(0,13-1,59)$ & $1,40 * *(1,09-1,82)$ & $0,87(0,45-1,67)$ & $1,63(0,63-4,20)$ \\
\hline \multicolumn{5}{|c|}{ RP (IC95\%) ajustadas por idade, sexo e escolaridade } \\
\hline Plano privado & 1,00 & 1,00 & 1,00 & 1,00 \\
\hline ESF & $0,46 * *(0,22-0,95)$ & $1,00(0,78-1,29)$ & $0,79(0,50-1,25)$ & $1,13(0,49-2,63)$ \\
\hline UBS "tradicional" & $0,51(0,14-1,84)$ & $1,35 * \star(1,04-1,77)$ & $0,92(0,48-1,77)$ & $1,54(0,60-3,97)$ \\
\hline Número de entrevistados & 370 & 370 & 370 & 370 \\
\hline
\end{tabular}

ESF: Estratégia Saúde da Família; IC95\%: intervalo de 95\% de confiança; RP: razões de prevalência; UBS: unidade básica de saúde.

Nota: as RPs e respectivos IC95\% foram estimados por meio de regressão de Poisson com variância robusta, considerando-se os parâmetros amostrais.

* Teste qui-quadrado para diferenças entre as fontes de atenção;

$\star * \quad p<0,05$.

incluem a realização de pelo menos uma consulta médica anual e a dosagem da glicemia duas ou mais vezes no ano, assim como a aferição da pressão arterial e do colesterol pelo menos uma vez no ano ${ }^{8}$. As informações obtidas no 20 Inquérito de Saúde de Adultos da Região Metropolitana de Belo Horizonte permitiram estimar essas metas na população do estudo, mas foi feita uma adaptação porque as informações coletadas contemplavam a realização de pelo menos uma dosagem de glicemia no último ano, sem especificação do número de aferições realizadas. Além dos indicadores anteriormente mencionados, na presente análise, foi considerada a existência de profissional ou serviços de referência, uma vez que existem evidências de que este vínculo favorece a adesão às recomendações dos profissionais de saúde, entre outros efeitos positivos 18. Esta análise também contemplou o consumo de medicamentos de uso regular e o relato de dificuldades para obtê-los. Os resultados mostraram que não existem diferenças significantes no desempenho dos indicadores mencionados entre indivíduos com diagnóstico de diabetes residentes em áreas cobertas pela ESF ou em áreas cobertas pela USB “tradicional”, em comparação aos afiliados a plano privado de saúde no Município de Belo Horizonte.
Com relação ao desempenho dos atributos da atenção primária, os resultados mostraram diferenças importantes entre as fontes de atenção, sobretudo no primeiro contato e no acesso. Entre os usuários exclusivos do SUS (residentes ou não em áreas cobertas pela ESF), a proporção dos que precisavam consultar um generalista para ser encaminhado a um especialista foi duas vezes maior do que entre afiliados a plano privado de saúde. Essa diferença é explicada pela organização do sistema de saúde 19 .

Estudos anteriores realizados em duas cidades brasileiras mostraram que o acesso à consulta médica é um dos atributos com o pior desempenho no sistema público de saúde 20,21. Os resultados deste trabalho são consistentes com essas observações, indicando que os usuários exclusivos do SUS são $60 \%$ mais propensos a terem dificuldades para a obtenção de consultas, em comparação aos seus pares afiliados a plano privado de saúde, independentemente da idade, do sexo e do nível de escolaridade. Os atributos da integralidade, da longitudinalidade e do foco familiar apresentaram pior desempenho entre usuários exclusivos do SUS residentes em áreas cobertas pela ESF, em comparação aos afiliados a plano privado de saúde, mas as diferenças entre estes dois grupos - embora estatisticamente 
significantes - foram menos expressivas do que as observadas nos atributos primeiro contato e acesso. O desempenho dos atributos da integralidade, da longitudinalidade e do foco familiar não diferiu significativamente entre residentes em áreas cobertas pela UBS “tradicional” e afiliados a plano privado de saúde, mas este resultado deve-se provavelmente ao pequeno número de pessoas com a doença na amostra de residentes nas áreas cobertas pela UBS "tradicional”.

O Ministério da Saúde reforça o processo educativo na atenção primária em saúde, de forma a melhorar os comportamentos em saúde dos indivíduos com diabetes ${ }^{8}$. Os comportamentos em saúde de pessoas com a doença são os resultados dos seus hábitos ao longo da vida e de mudanças em consequência do diagnóstico de diabetes. Estudos transversais, como é o caso da presente investigação, não permitem saber se os comportamentos atuais foram modificados em relação àqueles antes do diagnóstico médico. Contudo, quando as diversas fontes de atenção foram comparadas, observou-se que entre os cobertos pela ESF ou pela UBS “tradicional” a prevalência da inatividade física no lazer foi mais do que o dobro da observada entre afiliados a plano privado de saúde, mesmo após ajustes por idade, sexo e nível de escolaridade, sugerindo que as ações no âmbito da ESF não estão sendo suficientes para a promoção da atividade física na população de estudo.

Este trabalho tem vantagens e limitações. As principais vantagens são o tamanho da amostra e a sua representatividade da população elegível; o local do estudo (a terceira maior cidade brasileira) e a existência de informações que permitem, por meio de georreferenciamento, localizar

\section{Colaboradores}

Todos os autores participaram de todas as etapas de produção do artigo. os domicílios em áreas cobertas ou não pela ESF. Outra vantagem do estudo é a análise de atributos da atenção primária para cuidados dos indivíduos com diabetes 8 , tratando-se - ao nosso conhecimento - do primeiro realizado entre diabéticos residentes na comunidade. Por outro lado, o estudo foi baseado em informações obtidas por meio de inquérito de saúde não delineado para ser representativo da população com diabetes na faixa etária elegível. Assim, vários indicadores de desempenho do cuidado ao paciente com diabetes 8 não puderam ser investigados. Outra limitação do trabalho é a ausência de medidas biológicas, impossibilitando a mensuração de parâmetros de controle da doença, tais como o nível da glicemia, aferições antropométricas e da pressão arterial, entre outros. Também é importante lembrar que o nosso estudo foi baseado no diabetes autorreferido, e estima-se que cerca da metade dos diabéticos residentes na comunidade desconhece ter a doença 8,22. Assim, as prevalências de diabetes autorreferido encontradas na presente investigação devem estar subestimadas e nossas inferências são baseadas nos moradores que sabem ter a doença.

O diabetes é considerado uma "condição sensível à atenção primária”, ou seja, uma condição de saúde cujo manejo adequado neste nível de atenção pode evitar hospitalizações e óbitos por complicações da doença 11. Os resultados deste trabalho apontam dois principais desafios para o SUS no manejo dos pacientes com diabetes no Município de Belo Horizonte: (1) realizar ações que sejam mais efetivas para a promoção da atividade física; e (2) reorganizar-se para melhorar o desempenho da atenção primária, sobretudo no acesso às consultas médicas.
Agradecimentos

O 2 o Inquérito de Saúde de Adultos da Região Metropolitana de Belo Horizonte foi financiado pelo Departamento de Atenção Básica, Secretaria de Assistência à Saúde, Ministério da Saúde. Os autores agradecem a Breno Dayrell Bretas Alvarenga Bastos, bioestatístico bolsista da Fiocruz Minas, pela cuidadosa preparação do banco de dados para análise, e a Walter Luiz Batista Ferraz, da Secretaria Municipal de Saúde de Belo Horizonte, pelo georreferenciamento dos domicílios participantes da pesquisa. 


\section{Referências}

1. United Nations. General Assembly. Prevention and control of non-communicable diseases. Report of the Secretary-General. http://www.un.org/en/ga/ ncdmeeting2011/documents.shtml (acessado em $15 / \operatorname{Jan} / 2015)$.

2. World Health Organization. Global health risks: mortality and burden of disease attributable to selected major risks. http://www.who.int/healthinfo/global_burden_disease/GlobalHealthRisks_re port_full.pdf (acessado em 15/Jan/2015).

3. World Health Organization. Global status report on non-communicable diseases. http://www.who. int/nmh/publications/ncd_report_full_en.pdf (acessado em 15/Jan/2015).

4. Bertoldi AD, Kanavos P, França GV, Carraro A, Tejada CA, Hallal PC, et al. Epidemiology, management, complications and costs associated with type 2 diabetes in Brazil: a comprehensive literature review. Global Health 2013; 9:62.

5. Sartorelli DS, Franco LJ. Tendências do diabetes mellitus no Brasil: o papel da transição nutricional. Cad Saúde Pública 2003; 19 Suppl 1:S29-36.

6. Duncan BB, Stevens A, Schmidt MI. Mortalidade por doenças crônicas no Brasil: situação em 2010 e tendências de 1991 a 2010. In: Departamento de Análise de Situação de Saúde, Secretaria de Vigilância em Saúde, Ministério da Saúde, organizador. Saúde Brasil 2011. Brasília: Editora do Ministério da Saúde; 2012. p. 93-104.

7. Lima-Costa MF, Stevens A, Duncan B. Mortalidade entre idosos no Brasil: tendências em 20 anos (1991 a 2010). In: Departamento de Análise de Situação de Saúde, Secretaria de Vigilância em Saúde, Ministério da Saúde, organizador. Saúde Brasil 2011. Brasília: Editora do Ministério da Saúde; 2012. p. 209-26.

8. Departamento de Atenção Básica, Secretaria de Atenção à Saúde, Ministério da Saúde. Estratégias para o cuidado da pessoa com doença crônica: diabetes mellitus. Brasília: Ministério da Saúde; 2013. (Cadernos de Atenção Básica, 36).

9. Gusso G, Lopes JMC. Tratado de medicina de família e comunidade. v. 2. São Paulo: Editora Artmed; 2012.

10. Rosa RS. Diabetes mellitus: magnitude das hospitalizações na rede pública do Brasil, 1999-2001. Epidemiol Serv Saúde 2008; 17:131-4.

11. Alfradique ME, Bonolo PF, Dourado I, Lima-Costa MF, Macinko J, Mendonça CS, et al. Internações por condições sensíveis à atenção primária: a construção da lista brasileira como ferramenta para medir o desempenho do sistema de saúde (Projeto ICSAP - Brasil). Cad Saúde Pública 2009; 25:1337-49.
12. Starfield B. Primary care: balancing health needs, services and technology. New York: Oxford University Press; 1998.

13. Paim J, Travassos C, Almeida C, Bahia L, Macinko J. The Brazilian health system: history, advances and challenges. Lancet 2011; 377:1778-97.

14. Panarotto D, Träsel HA, Oliveira MS, Gravina LB, Teles AR. Controle glicêmico de pacientes diabéticos tipo 2 nos serviços público e privado de saúde. Arq Bras Endrocrinol Metabol 2009; 53:733-74.

15. Instituto Brasileiro de Geografia e Estatística. Indicadores sociais municipais: uma análise dos resultados do universo do Censo Demográfico 2010. Rio de Janeiro: Instituto Brasileiro de Geografia e Estatística; 2011. (Estudos e Pesquisas. Informação Demográfica e Socioeconômica, 28).

16. Lima-Costa MF, Turci M, Macinko J. Estratégia Saúde da Família em comparação a outras fontes de atenção: indicadores de uso e qualidade dos serviços de saúde em Belo Horizonte, Minas Gerais, Brasil. Cad Saúde Pública 2013; 29:1370-80.

17. Long JS, Freese J. Regression models for categorical dependent variables using stata. 2nd Ed. College Station: Stata Press; 2006.

18. Cabana MD, Jee SH. Does continuity of care improve patient outcomes? J Fam Pract 2004; 53: 974-80.

19. Almeida PF, Giovanella L, Mendonça MH, Escorel S. Desafios à coordenação dos cuidados em saúde: estratégias de integração entre níveis assistenciais em grandes centros urbanos. Cad Saúde Pública 2010; 26:286-98.

20. Araújo LU, Gama ZA, Nascimento FL, Oliveira HF, Azevedo WM, Almeida Júnior HJ. Avaliação da qualidade da atenção primária à saúde sob a perspectiva do idoso. Ciênc Saúde Coletiva 2014; 19:3521-32.

21. Castro RC, Knauth DR, Harzheim E, Hauser L, Duncan BB. Avaliação da qualidade da atenção primária pelos profissionais de saúde: comparação entre diferentes tipos de serviços. Cad Saúde Pública 2012; 28:1772-84.

22. Lima-Costa MF, Peixoto SWV, Firmo JOA, Uchoa ME. Validade do diabetes auto-referido e seus determinantes: evidências do Projeto Bambuí. Rev Saúde Pública 2007; 41:947-53. 


\section{Abstract}

This study compared the use of health services and medication, screening test coverage, characteristics of primary care, and health behaviors in 370 diabetics 45 years or older according to sources of care: private health policyholders regardless of place of residence; residents in areas covered by the Family Health Strategy; and individuals covered by a "traditional" Primary Care Unit. The study used data from the $2^{\text {nd }}$ Adult Health Survey in Belo Horizonte Metropolitan Region (2010), Minas Gerais State, Brazil. Use of health services, use of medication, coverage of follow-up tests, alcohol abuse, and smoking did not differ significantly according to source of care. Prevalence rates for insufficient leisure-time physical exercise and sedentary lifestyle were higher among individuals covered by the Family Health Strategy or "traditional" Primary Care Unit. The primary care characteristics (access, comprehensiveness, continuity, and family focus) performed better among individuals with private health insurance, even after adjusting for age, sex, and schooling. The study identified two main challenges for the Brazilian Unified National Health System in managing patients with diabetes in the city of Belo Horizonte: the promotion of physical exercise and reorganization to improve performance in primary care, especially in access to medical appointments.

Diabetes Mellitus; Health Services; Family Health; Primary Health Care

\section{Resumen}

El objetivo del estudio fue comparar el uso de servicios de salud, el uso de medicamentos, la cobertura de exámenes de rastreo, los atributos de atención primaria en salud y los comportamientos en salud de individuos $(n=370)$ con 45 años o más, con un diagnóstico médico de diabetes, según las fuentes de atención en salud, así definidas: afiliados a plan privado de salud, independientemente del lugar de residencia; residentes en áreas cubiertas por la Estrategia Salud de la Familia (ESF); cubiertos por la unidad básica de salud (UBS) "tradicional". El estudio utilizó los datos de la 2a Encuesta de Salud de Adultos de la Región Metropolitana de Belo Horizonte (2010). Los indicadores de uso de servicios de salud, el uso de medicamentos, la cobertura de exámenes de seguimiento, el consumo abusivo de alcohol y el tabaquismo actual no difirieron significativamente entre las fuentes de atención. Las prevalencias de la práctica insuficiente de ejercicios físicos en el tiempo libre y del sedentarismo en lo cotidiano fueron mayores entre los cubiertos por la ESFo UBS "tradicional". Los atributos de la atención primaria en salud (acceso, integralidad, longitudinalidad y foco familiar) presentaron el mejor desempeño entre los afiliados al plan privado de salud, incluso tras el ajuste por edad, sexo y escolaridad. Los resultados de la investigación apuntan dos principales desafios para el Sistema Único de Salud en el manejo de los pacientes con diabetes en el municipio de Belo Horizonte, Minas Gerais, Brasil: la realización de acciones más efectivas para la promoción de la actividad física y la necesidad de reorganización con vistas a una mejoría en el desempeño de la atención primaria, sobre todo en el acceso a las consultas médicas.

Diabetes Mellitus; Servicios de Salud; Salud de la Familia; Atención Primaria de Salud
Recebido em 02/Fev/2015

Versão final reapresentada em 17/Fev/2016

Aprovado em 11/Mar/2016 